Voix et Images

\title{
De quelques influences possibles sur la vision du monde de Gabrielle Roy : George Wilkinson et Henri Girard
}

\section{Ben-Z. Shek}

Volume 14, numéro 3 (42), printemps 1989

Gabrielle Roy

URI : https://id.erudit.org/iderudit/200798ar

DOI : https://doi.org/10.7202/200798ar

Aller au sommaire du numéro

Éditeur(s)

Université du Québec à Montréal

ISSN

0318-9201 (imprimé)

1705-933X (numérique)

Découvrir la revue

Citer cet article

Shek, B.-Z. (1989). De quelques influences possibles sur la vision du monde de Gabrielle Roy : George Wilkinson et Henri Girard. Voix et Images, 14(3),

437-452. https://doi.org/10.7202/200798ar d'utilisation que vous pouvez consulter en ligne. 


\title{
De quelques influences possibles sur la vision du monde de Gabrielle Roy: George Wilkinson et Henri Girard
}

\author{
par Ben-Z. Shek, Université de Toronto
}

Le premier roman de Gabrielle Roy, Bonheur d'occasion', constitue une charge habilement esthétisée contre le système capitaliste qui ne pouvait «résoudre» la crise économique des années trente qu'au moyen du «salut» ironique de la guerre. Pour Albert Le Grand, ce roman constituait la condamnation d'une société qui trahit ses pauvres ${ }^{2}$, tandis qu'André Brochu est allé encore plus loin, y voyant l'expression d' une prise de conscience tragique, presque révolutionnaire de la misère et des conflits sociaux qui rongeaient un quartier canadien-français $[\ldots]^{3}$. Nous avons nous-même analysé en détail la portée contestataire, progressiste de cette cuvre ${ }^{4}$, que Gilles Marcotte, quant à lui, avait présentée dans un texte intitulé «En relisant Bonheur d'occasion», comme une fresque sociale dont l'ampleur et la profondeur sont inégalées au Canada français $[\ldots]^{5}$.

Dans son discours de réception à la Société royale du Canada, Gabrielle Roy a offert un commentaire combien poignant de son premier livre sous la forme d'une mise à jour de ses personnages. Intitulé «Bonheur d'occasion aujourd'hui» ${ }^{6}$, le discours résume l'on ne peut mieux le côté revendicateur de son roman. Et l'un de ses personnages ultérieurs, le missionnaire André Le Bonniec, dans la Montagne secrète, le fait peut-être pour l'œuvre entière quand il déclare: Des protestataires [...] comment se fait-il que tout ce qui se fait de plus beau dans ce monde soit un acte de protestation. Créer [...] n'est-ce pas de toute son âme protester? (MS, p. 131) À travers toute son cuvre, y compris dans les textes où l'auteure se tourne davantage à l'intérieur d'elle-même que vers la société ambiante, il y a une nette identification de l'instance narrative

1 Première édition: Montréal, Société des Editions Pascal, 1945, 2 vols.

2 «Gabrielle Roy ou l'être partage»», Etudes françalses, vol. I, no 2, juin 1965, p. 45.

3 «Thèmes et structures de Bonheur d'occasion», Ecrits du Canada français, XXII, 1966, p. 165.

4 Social Realism in the French-Canadian Novel, Montréal, Harvest House, 1977, p. 65-111.

5 Gilles Marcotte, Une littérature qui se fait, Montréal, Éditions HMH, 1962, p. 39. 40 (Constantes, no 2).

6 Reproduit dans le Bulletin des agriculteurs, vol. XLIV, no 4, janvier 1948, p. 6-7, 20-23, et repris dans Fragiles Lumières de la terre, 1978, p. 159-175. 
avec le peuple besognant 7 . Identification résumée peut-être par la petite Christine de la Route d'Altamont qui, rêvant que sa grand-mère remplace Dieu au ciel et règne sagement, avec justice, pense: Or le pauvre monde sur terre s'en trouvait bien! (p. 31)

Et vers la fin de sa trajectoire terrestre, dans sa somme, la Détresse et l'enchantement qui, posthume, a couronné l'œuvre entière, Gabrielle Roy a encore une fois manifesté sa vision du monde progressiste et démocratique dans les passages traitant de sa rupture avec Stephen, Canadien d'origine ukrainienne, anti-communiste et fascisant, pour qui elle a quand même ressenti une attraction physique et affective des plus fortes. Elle fait de même dans les passages où elle découvre des réfugiés républicains fuyant les troupes de Franco pendant la guerre civile d'Espagne, à un moment où, au Canada français, bien des élites sont tombées dans le piège du corporatisme et d'autres totalitarismes.

Vers la toute fin de son autobiographie, justement, Gabrielle Roy raconte la genèse de ce qui deviendra Bonheur d'occasion. Un certain soir d'avril 1939, où elle longeait le canal Lachine, comparant ses premiers billets publiés dans le Jour - des banalités - et la grande tâche qu'elle entreprendrait sous peu et qui deviendrait son premier roman, elle dit avoir fait face à la peur: Mais $d u$ moins alors je serais happée entière par le sujet, aidée et soutenue par tout ce que j'aurais acquis de ressources, de connaissances de l'humain et par la solidarité avec mon peuple retrouvé [...]. (DE, p. 504-505)

La question qui nous intéresse est la suivante: est-ce qu'une partie de ces connaissances de l' humain repose sur ses rapports avec George Wilkinson et Henri Girard, entre 1939 et la publication de Bonheur d'occasion en 1945? Et si oui, comment ces deux personnages ont-ils influencé cette vision du monde?

Revenons pour un moment à la Détresse et l'enchantement. C'est là aussi, vers la fin, que l'auteure nous décrit son premier logement près du terminus d'autobus, au centre-ville de Montréal, puis son second logement, chez une certaine «Miss McLean»: Je montai la longue côte d'Atwater. Je pris la rue Dorchester et me trouvai a passer sans le savoir devant la maison où je viendrais bientôt prendre une chambre (p. 503). C'est dans cette maison-là, décrite notamment dans How I Found the People of Saint-Henri ${ }^{8}$, et qui se trouvait à mi-chemin entre Westmount et Saint-Henri, les deux principaux espaces qui modèlent Bonheur d'occasion, que, pendant une période de temps indéterminée au début de la Seconde Guerre mondiale, Gabrielle Roy a vécu avec un certain George Wilkinson.

$7 \quad$ Voir Social Realism..., p. 66.

8 Plaquette publiée par la Literary Guild of the United States, 1947. 
Mes informations sur cette liaison proviennent du regretté criminologue québécois Stephen Cumas ${ }^{9}$, proche ami de Wilkinson et qui a fréquenté le couple à cette époque. Selon Cumas, c'est Wilkinson qui avait fait entrer Gabrielle Roy à l'hebdomadaire le Jour, où elle publia trente chroniques entre le 6 mai 1939 et le 16 mars 1940. Les liens entre Wilkinson et Roy se seraient donc tissés peu après le retour d'Europe de la romancière en avril 1939. Nous n'avons pas pu établir la durée de cette liaison, mais $M$. Cumas nous a raconté que Gabrielle Roy l'avait rompue à cause du comportement paranoïaque de Wilkinson, qui craignait que quelqu'un ne veuille l'empoisonner ${ }^{10}$.

Mais qui était George Wilkinson et comment aurait-il pu influer sur la démarche idéologique de Gabrielle Roy? C'était un type original et très coloré, né à Saint-Jean, Terre-Neuve, en 1897 ou 1898, donc de douze ou treize ans l'aîné de Gabrielle Roy ${ }^{11}$. Wilkinson, tout jeune encore, commença une carrière d'apprentipasteur dans l'Église méthodiste de Terre-Neuve en 1915, au village de SeldomCome-By. En 1917 et 1918, il continuait à desservir de petites communautés terre-neuviennes telles Blackhead et Millertown. Puis, en 1919, il partit pour Montréal où il passa sept ans. Il y fit des études au Wesleyan Theological College de l'université McGill entre 1919 et 1925, et fut ordonné pasteur de l'Église unie avec un diplôme en théologie en 1926, un an après que cette confession eut

9 Stephen Cumas (né Koumoutsidis) fut directeur de la John Howard Society du Québec, puis membre de la Commission fédérale des libérations conditionnelles. Il est décédé le 2 juin 1986, avant que nous ne puissions obtenir de lui de plus amples renseignements sur la vie de George Wilkinson à Montréal.

10 Nous verrons plus loin une source possible de ce comportement dans les expériences de Wilkinson à Terre-Neuve avant qu'il ne s'établisse à Montréal. L'on croit qu'il est mort de faim vers 1952 à Oka, où il vivait avec les Amérindiens, à cause de cette peur d'être empoisonné, mais nous n'avons pu vérifier ces faits. Dans une conversation à Montréal le 10 décembre 1988, la fille de Stephen Cumas, Pennie Cowie, exprima l'avis que c'était plutôt Wilkinson qui avait rompu avec Gabrielle Roy, ajoutant qu'ils étaient fiancés à l'époque, selon les dires de ses parents. $\mathbf{M}^{m}$ Cowie se rappelle les visites de Wilkinson (Il était grand et blond) à la maison familiale des Cumas à Cartierville, entre 1949 et 1951 . Wilkinson, qui jouait du piano, l'encourageait dans ses leçons, bien que $\mathbf{M}^{\mathrm{me}}$ Cowie jou[ât] mal. Selon elle, Wilkinson travaillait alors comme garçon d'ascenseur dans un des grands magasins du centre-ville montréalais. La mère de Mmo Cowie, Helen Gardner (née Khouri), première femme de Stephen Cumas, nous raconta, le 12 décembre 1988, qu'elle avait rencontré Wilkinson peu après son mariage avec M. Cumas en 1935. Le Terre-Neuvien fréquentait le restaurant The Briton à l'angle des rues Guy et Sainte-Catherine, que gérait son mari. C'était un homme gentil et chaleureux, mais timide. En 1952, il était au bout de son rouleau et ne mangeait que des dattes, selon $\mathbf{M}^{\text {mo }}$ Gardner. Elle ajouta que Wilkinson voulait briser le monopole de la compagnie Hacquet, qui importait le poisson de Terre-Neuve, pour venir en aide aux pêcheurs de l'ile, et avait fait appel pour cela à son père, Aboo Samra Khouri, importateur d'origine libanaise, mais l'affaire n'aboutit pas.

$11 \mathrm{M}$. Wilkinson avait trente-huit ans lors de son procès devant la Cour suprême de Terre-Neuve en mai 1935, procès dont nous parlerons plus loin. Les renseignements sur la vie de Wilkinson à Terre-Neuve et sur ses études universitaires nous ont été fournis par M. Rick Stapleton, archiviste de l'Église unie du Canada, et sont fondés sur deux documents préparés par M. Douglas Walkington: «Methodist Ministers» et «United Church Ministers». 
émergé de la fusion des Églises méthodiste, presbytérienne et congrégationaliste. Wilkinson possédait aussi une maîtrise, vraisemblablement en philosophie. En plus, il fit des études universitaires de premier cycle au Vermont et de troisième cycle à Harvard, ces dernières entre 1928 et 1932. Il passa une année de pastorat à Comerbrook, Terre-Neuve, en 1927, avant son stage à la célèbre université américaine. Il ne semble pas, pourtant, qu'il ait jamais terminé son doctorat. Pendant un certain temps, il enseigna dans une école de Westmount, vers 1932.

Entre 1932 et 1934, Wilkinson travailla à Montréal comme représentant d'une maison de produits pharmaceutiques de Terre-Neuve, ayant démissionné du pastorat en 1933 ou 193412. Pendant ses loisirs, il participait à la collecte de vêtements d'occasion que l'on envoyait aux miséreux de Saint-Jean, et parlait à la radio de la situation socio-économique de Terre-Neuve. Wilkinson rentra à Saint-Jean en avril 1934, à cause d'un conflit avec une compagnie de bateaux à vapeur au sujet d'une commande de produits pharmaceutiques dont il était responsable. Il ne tarda pas à s'engager dans l'action sociale, surtout dans le mouvement des chômeurs. Wilkinson dispensa des médicaments aux victimes d'une épidémie de coqueluche en décembre 1934 et enseigna, en bénévole, trois soirs par semaine, aux enfants pauvres, dans une école du quartier de la Battery à Saint-Jean.

Wilkinson devint vite un des leaders du comité des chômeurs de Saint-Jean. Bien qu'il ne fût jamais membre d'un mouvement politique ou d'un syndicat, il pensa nécessaire de s'engager en faveur des sans-travail, for the sole purpose of helping his distressed fellow countrymen ${ }^{13}$. Il commença à prendre la parole lors de grandes manifestations qui réunissaient dans la capitale terre-neuvienne entre deux et cinq mille citoyens, en soutien aux chômeurs, aux familles pauvres et aux vétérans de la Première Guerre mondiale qui, eux aussi, souffraient énormément de la situation économique désastreuse.

En 1934, le gouvernement britannique suspendit la constitution de sa colonie de Terre-Neuve et nomma une commission gouvernementale dont tous les membres étaient des Britanniques, pour gérer les affaires de l'île. Wilkinson et ses camarades du comité des chômeurs se heurtèrent à la commission quand ils demandèrent, au début de mai 1935, que l'on remplace le bien-être social - l'on versait à l'époque 1,60 \$ par mois aux chômeurs célibataires - par du travail rémunéré. Le comité voulait en outre jouer un rôle dans l'inscription des chômeurs en vue d'obtenir du travail dans des projets que la commission, sous les pressions, avait promis de créer. Lors d'une grande manifestation, le 10 mai 1935, devant les édifices du Colonial Building dans la capitale, Wilkinson et

12 Les renseignements qui suivent nous ont été foumis par le professeur James Overton, sociologue de la Memorial University de Saint-Jean, Terre-Neuve, sous la forme de coupures de quotidiens, ainsi que de rapports policiers tirés des «Provincial Archives of Newfoundland and Labrador». Nous tenons à le remercier chaleureusement.

13 «Defence in Riot Trial», Evening Chronicle, Saint-Jean, 30 mai 1935, p. 13. Traduction: [...] dans le seul but d'aider ses concitoyens en détresse. 
quelques autres leaders des sans-travail furent matraqués par la police. Il reçut des coups à la tête et aux mains en essayant de se protéger de trois policiers; il décrivit ce qui s'était passé en ces termes: Like Jesus, the marks are on my hands and feet. ${ }^{14}$ Un membre du comité déclara que l'attaque policière was like a slaughter at the icefields ${ }^{15}$.

Les chefs arrêtés furent accusés d'avoir causé une émeute et comparurent devant la Cour suprême le 27 mai 1935. Le 31 mai, quatre leaders du comité des chômeurs, dont Wilkinson, furent trouvés non coupables et libérés, aux applaudissements des personnes présentes à l'intérieur de l'édifice, puis d'un grand nombre de chômeurs qui attendaient dehors ${ }^{16}$. Pendant le procès, l'on cita des discours de l'un d'eux, Pierce Power, qui aurait dit: Working people are the quietest in the world. They are starved, murdered and done everything with. They are innocent, but there is a limit to their innocence. ${ }^{17}$ Et aussi Wilkinson, qui aurait décrit les logements pitoyables où vivaient la plupart des chômeurs, et exigé que l'on taxe les propriétaires absents. Dans la ville de Grand Falls, dit-il, les ouvriers étaient à demi morts de faim, begging with shoes that were no longer shoes but pads of leather sown together ${ }^{18}$. Un autre leader, Joseph Milley, aurait ironise: The budget is balanced, the bondholders are paid and you can go to hell and starve. 19

Avant et après la grande manifestation du 10 mai 1935, Wilkinson fut régulièrement suivi par des agents secrets du Constabulary Department de Saint-Jean, en tant que political agitator ${ }^{20}$. Dans une plaquette qu'il avait rédigée, et que la police repéra, il décrivait les Terre-Neuviens comme a starving, diseased, oppressed, dying people, et en appela aux chômeurs: Only the truth can make us free and keep us free. Our words must of necessity be simple, direct, honest and fearless. 21 Il fustigea la commission gouvemementale en ces

14 Ibid. Traduction: Comme Jésus, les marques sont sur mes mains et sur mes pieds.

15 Ibid., 31 mai 1935, p. 6. Traduction: [...] était comme un massacre sur les champs de glace. Ces paroles sont celles de Pierce Power, l'un des chefs du mouvement des chômeurs.

16 Les accusés avaient insisté pour dire que les pierres avaient été lancées après et non avant l'attaque à la matraque.

17 «Riot Trial», Evening Chronicle, 28 mai 1935, p. 5. Traduction: La classe ouvrière est la plus tranquille au monde. Elle est affamée, assassinée et meurtrie de toutes les façons. Elle est innocente, mais il y a une limite à son innocence.

18 Ibid. Traduction: [...] mendiant avec des chaussures qui n'étaient plus des chaussures mais des morceaux de cuir cousus ensemble.

19 Ibid. Traduction: Le budget est équilibré, les porteurs d'obligations sont payés et vous pouvez aller en enfer et $y$ crever.

20 Constable Harry Glover, rapport au chef de police de Saint-Jean, P.J. O'Neill, daté du 21 mars 1935 (Provincial Archives of Newfoundland and Labrador, GN 131, Box 155, File 24).

21 «What about it, Newfoundlanders?» by George Wilkinson, M.A., member of Committee, Unemployed, St. John's, s.d., quatre pages ronéotypées, mises en appendice au rapport du constable Harry Glover (voir note précédente). Traduction: [...] une population affamée, malade, opprimée, mourante. [...] Seule la vérité peut nous rendre et nous garder libres. Par nécessité, nos mots doivent être simples, directs, honnêtes et braves. 
termes: $[. .$.$] as a government your presence would not be tolerated one day$ among a people who had the faintest glimmerings of what real democracy means. Your very presence is nothing short of an invasion. 22

Même après leur procès, Wilkinson et les autres membres du comité des chômeurs continuèrent à militer. Lors d'une manifestation à Beck's Cove, dans la région de Saint-Jean, le 19 août 1935, Herbert Saunders, l'un des leaders du comité, déclara: Wilkinson has gone away tonight - his head beaten silly with billies. ${ }^{23}$ Pendant une réunion du 26 août, selon un rapport policier, Saunders se référa à une attaque policière aux «Colonial Grounds» qui aurait eu lieu en juin et où il reprocha aux chômeurs d'avoir abandonné leurs chefs, dont Wilkinson, à la police 24 . En tout cas, il semble que Wilkinson ait quitté Terre-Neuve pour Montréal le 19 août 1935.

Ce qu'il y fait précisément entre 1935 et 1939, où commencent à paraitre ses textes dans le Jour, n'est pas très clair. Nous savons qu'il a enseigné de nouveau dans la région montréalaise, dans une école de langue anglaise, et aussi qu'il a trouvé abri et nourriture au restaurant de la famille du criminologue Stephen Cumas, rue Sainte-Catherine, pendant les dernières années de la crise ${ }^{25}$.

En tout cas, George Wilkinson signa quatre textes dans le Jour, entre le 19 août 1939 et le 16 novembre 1940, et fut cité comme source principale d'un cinquième texte, «Terre-Neuve et le Canada», qui parut le 7 décembre 1940 (l'on aura remarqué que c'est à peu près à cette période - entre le 6 mai 1939 et le 16 mars 1940 - que Gabrielle Roy publia ses chroniques dans le même hebdomadaire). Dans son premier article 26 , Wilkinson insiste sur le fait que son île natale constitue la clé de la défense de tout le continent nord-américain, thème qu'il reprendra à plusieurs reprises, et qu'il liera aux conditions d'extrême pauvreté qui y prévalent. Il note que le nombre des bénéficiaires de l'une ou l'autre des formes d'aide sociale à Terre-Neuve est passé d'environ

22 Ibid. Traduction: [...] comme gouvernement, votre présence ne serait pas tolérée un seul jour par un peuple qui aurait la moindre idée de ce qu'est la vraie démocratie. Votre présence même n' est rien de moins qu' une invasion.

23 Cité dans M.P. Mahoney, rapport au chef de police, P.J. O'Neill («Re: Unemployed Meeting Tonight in Beck's Cove», daté du 19 aoû́t 1935, p. 11). Ces blessures-là peuvent être la cause de la paranoïa ultérieure de Wilkinson. Traduction: Wilkinson est parti ce soir - la tête meurtrie par les matraques.

24 They ran away and left him [Pierce Power] to be clubbed to death, myself, Milley and Wilkinson, cité dans M.R. Mahoney, Detective Constable, rapport au chef de police, P.J. O'Neill («Re: Unemployed Meeting Tonight», daté du 26 août 1935). II n'est pas clair si juin voulait dire mai dans ce rapport, ou s'il s'agissait effectivement d'une seconde attaque à la matraque après celle du 10 mai 1935 . Traduction: Ils se sont enfuis et ont laissé Pierce Power se faire battre à mort, moi-même, Milley et Wilkinson.

25 C'est Phyllis Cumas qui nous a communiqué ce renseignement. Wilkinson faisait partie d'un groupe d'intellectuels et d'artistes qui se réunissaient là au cours de cette période. Voir la note 10 pour plus de renseignements.

26 «De quel droit? Opinions d'un Terre-Neuvien», le Jour, 19 août 1939, p. 4. Toutes les autres citations de ce paragraphe sont tirées du même article. 
$50 \%$ de la population en 1938 à $75 \%$ en 1939, soit de 140000 d 210000 en douze mois, sur une population de 285000 . De nouveau, il attaque la commission gouvernementale, la qualifiant de ploutocratie déguisée en aristocratie, au moment ou l'achat possible des honneurs devient chose publique. Il cite un ami qui, rentré de Terre-Neuve, déclarait que si les Terre-Neuviens étaient sérieusement armés, il seraient à l' heure actuelle en pleine révolte, et conclut ce premier texte ainsi:

L'homme d'affaires a, en politique, intronisé le Veau d'or, en a organisé le culte fatal et en a contaminé l'humanité entière. L'Homo sapiens, hypnotisé par l'ardent métal, a perdu le sens de la vie, la conception des valeurs. La misère de Terre-Neuve en est la preuve convaincante. Il est temps qu'elle cesse. Elle ne peut cesser qu'en autant que notre réorganisation sociale saura contrôler les puissances d'argent, sinon ces puissances nous contrôleront jusqu'd l'extinction rapide et complète de l' espèce humaine.

Dans son deuxième texte pour le Jour, publié le 2 septembre 1939, Wilkinson se moque des Britanniques qui ont aboli la constitution de TerreNeuve sous le prétexte que Nous, Terre-Neuviens, [sommes] [...] amoraux, tachés de lèpre mentale, corrompus jusqu'd la moelle des os, jusqu'à la dernière cellule grise27. Dans un troisième article, cette fois en anglais, Wilkinson, tout en rappelant son rôle de critique constant du Royaume-Uni, en appelle quand même à une participation canadienne et terre-neuvienne à la guerre qui vient d'éclater: Neutrality is no safeguard from war today $[. . . J$ We are the first line of your defence. We are the spear-point of the defence, without which you might not sleep so soundly as you do now. ${ }^{28}$

Dans un demier texte, «Terre-Neuve», paru le 16 novembre $1940^{29}$, Wilkinson polémique contre un certain $\mathrm{F}$. Butcher, qui a signé un article dans le Canadian Forum le mois précédent, intitulé «The Inertia of Newfoundland». Wilkinson propose de remplacer inertia par inanition et continue:

La putréfaction fait des ravages depuis longtemps: de mes propres yeux j'ai pu m' en rendre compte et $j^{\prime}$ ai tremblé à la vue de l'horrible squelette qui se cache derrière des souffrances terribles. Après tout, la

27 «Opinion d'un Terre-Neuvien», le Jour, 2 septembre 1939, p. 6.

28 «The Opinions of a Newfoundlander", le Jour, 16 septembre 1939, p. 5. Traduction: La neutralité n' est pas une garantie contre la guerre aujourd' hui [...] Nous sommes la première ligne de votre defense. Nous sommes le fer de lance de la défense, sans laquelle vous ne pourriez pas dormir aussi profondément que vous le faites maintenant. À côté de cet article, paraissait une notice annonçant une brochure écrite par Wilkinson, The Gordian Knot in Canada's Defence, et que l'on pouvait se procurer aux bureaux du Jour. L'auteur y prônait la Confédération entre TerreNeuve et le Canada, aussi bien que l'armement rapide pour faire face au fascisme. Nous n'avons pu retrouver cette brochure, ni à la Bibliothèque nationale du Canada, ni aux Archives nationales, ni à celles du ministère de la Défense nationale.

29 Le Jour, 16 novembre 1940 , p. 6 . Toutes les citations de ce paragraphe sont tirées de cet article. 
distribution d'un montant de 1,60 \$ par mois n'est pas plus faite pour une personne civilisée que ne le sont des sacs vides de farine pour fabriquer des jupons et des tabliers, des pantalons et des rideaux. ${ }^{30}$

Wilkinson raconte ensuite que:

Des écoliers a qui j'enseignais gratuitement furent exclus de la parade des enfants, lors du Jubilé d'Argent du Roi en 1935. Pourquoi? Parce qu' eux aussi n'étaient pas suffisamment vêtus pour suivre la parade dans les rues de la Vieille Colonie.

Se toumant vers le domaine de la santé, il ajoute: La tuberculose fait des ravages et le taux de mortalité est douze fois plus élevé que celui du Canada. Ensuite, il compare les salaires des experts, de 10000 \$ par an, au revenu moyen d' un père de famille de cinq personnes [qui] ne dépasse pas 110 \$ par an, et conclut: [...] les Terre-Neuviens sont destinés d toujours être traités comme des scieurs de bois ou des porteurs d'eau dans leur propre pays [...]. Enfin, dans un article anonyme, «Terre-Neuve et le Canada»31, accompagné d'une notice citant la documentation qui lui a servi de base, et où il est question de l'apport de $M$. George Wilkinson, un Terre-Neuvien bien placé pour être renseigné sur les vicissitudes de son pays, il est écrit: Il s'ensuit que les conditions générales de vie, déjà trop affectées à Terre-Neuve par la crise financière, y sont devenues de plus en plus difficiles. C'est ainsi que les enfants des pêcheurs de phoques de la baie Bonaventure ne peuvent plus fréquenter l'école parce qu' ils manquent de vêtements. ${ }^{32}$

N'est-il pas évident que les expériences de Wilkinson à Terre-Neuve, résumées dans ses textes du Jour, ont pu nourrir la future vision du monde de Gabrielle Roy, en aiguisant sa consciense sociale face à la crise, au chômage et à la misère inouïe qui en résultait? Ne trouve-t-on pas des parallèles entre maintes descriptions des chômeurs terre-neuviens, de leurs logis, vêtements, comportements, et certaines scènes frappantes de Bonheur d'occasion ${ }^{33}$ ? De

30 Et Wilkinson d'ajouter: Je me souviens d'un soir aux environs de minuit. Un vétéran se tenait sur le seuil de la porte, chez mon père. La faim et la fatigue lui donnaient un air sauvage et hideux; mais il parvint à formuler une demande: «Je meurs de faim». C'était malheureusement trop vrai. Son épouse et lui se chauffaient cet hiver-là de 1934-35 avec un baril de goudron l...] La porte [de leur maison] n'avait pas de peinture, les fenêtres n'avaient pas de vitres. Et cet homme possédait plusieurs médailles pour sa bravoure.

31 Sous la nubrique générale «Â travers l'actualité mondiale», le Jour, 7 décembre 1940, p. 4.

32 Pour une comparaison avec ces aspects dans Bonheur d'occasion, voir notre ouvrage Social Reallsm..., p. 68-79.

33 Ibid., p. 79-82. Le «salut» par la guerre dans Bonheur d'occasion semble être une sorte d'inversion ironique du manque de recrutement chez les chômeurs de TerreNeuve à la veille de la Seconde Guerre mondiale. Voir George Wilkinson, «Opinions of a Newfoundlander» (note 28, supra): Today England calls us as in 1914. We have been deprived of our vote, the control of government [...] It is no wonder that recruiting is so slow in the Island [...] The circumstances are so bad they cannot be worse; then why worry about the present for any future when there really is no 
plus, il y a un parallèle entre le soutien de Wilkinson à l'effort de guerre, face à la menace du fascisme, accompagné d'une critique sévère du système social tenu pour responsable de la crise, et l'enchevêtrement de ces deux aspects de l'actualité dans Bonheur d'occasion.

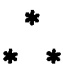

Nous nous sommes déjà référé, plus haut, aux chroniques publiées par Gabrielle Roy dans le Jour, à peu près au même moment où paraissaient les articles de Wilkinson, et au jugement sévère de la romancière sur ces textes de jeunesse. Une lecture des trente articles confirme en termes généraux l'appréciation de Gabrielle Roy. Néanmoins quelques textes sont intéressants: «Les logeuses de Montréal», le tout premier paru le 6 mai 1939 (p. 7); «Quelques jolis coins de Montréal», du 22 juillet de la même année (p. 2); «Noël chez les colons ukrainiens», du 30 décembre 1939 (p. 2); et surtout la seule nouvelle, «Nikolaï Suliz», du 3 février 1940 (p. 7), textes qui contiennent quelques éléments des futurs écrits. Située dans les environs de Winnipeg, l'action de la nouvelle traite à la fois d'une minorité ethno-culturelle et du chômage, ainsi que de l'ambiance industrielle, Gabrielle Roy y peignant un amas de toits gris et de hautes cheminées crachant la fumée dans le ciel froid.

Mais ce qui est sans doute plus important que les premiers essais d'écriture de Gabrielle Roy dans le Jour, c'est l'ambiance socio-politique, intellectuelle et artistique qui y régnait à l'époque où l'écrivaine y a fait son entrée, et dont les articles de Wilkinson étaient le reflet. Cette atmosphère est étudiée en détail dans le livre de Victor Teboul, le Jour: émergence du libéralisme moderne au Québec $^{34}$, qui se concentre sur les trois premières années de publication de l'hebdomadaire dirigé par Jean-Charles Harvey, à savoir entre le 16 décembre 1937 et le 5 octobre 1940. Ce périodique, qui s'est attiré les foudres et la censure épiscopales, a eu, selon Teboul, trois axes d'articulation; le combat idéologique, l'aire culturelle et la fonction littéraire (p. 20), et a été soutenu par divers groupes sociaux et politiques, y compris des gens de la gauche radicale (p. 38-39). Teboul montre l'ouverture du Jour aux moyens d' expression encore neufs au Québec à l'époque, comme le cinéma et la photographie, formes esthétiques que les journaux au style sévère de l'idéologie conservatrice comme le Devoir surveillent avec méfiance (p. 41). Il nomme, ensuite, plusieurs jeunes auteurs et artistes qui y ont signé des textes entre 1937 et 1940: Jean-Jules Richard, Gilles

future for such down-and-outs and near down-and-outs? (Traduction: Aujourd' hui, l'Angleterre nous appelle comme en 1914. Nous avons été privés de notre droit de vote, du contrôle du gouvernement [...]. Il n' est pas surprenant que le recrutement soit si lent dans l'Île $[$.... Les circonstances sont si défavorables qu'elles ne peuvent être pires; alors, pourquoi s'en faire maintenant pour l'avenir quand il n'y a pas d'avenir pour ces sans-le-sou et presque sans-le-sou?).

34 Montréal, Hurtubise HMH/Cahiers du Québec, 1984 (Communications). Toutes les citations tirées de cet ouvrage seront dorénavant suivies de la mention des pages entre parenthèses. 
Hénault, Jean-Paul Lemieux, Yves Thériault, Andrée Maillet, «Jean Narrache» (Émile Coderre), Jovette Bemier, Robert Choquette et le libraire Henri Tranquille. L'on pourrait ajouter Pierre Gélinas, Clément Marchand, Jean-Aubert Loranger et Berthelot Brunet. Louis Dantin, à partir de 1938, devient le collaborateur le plus important de ce périodique qu'il considérait comme l'unique refuge $[. .$.$] de la$ pensée indépendante, de l'honnête recherche de la vérité quelle qu'elle soit, des dernières libertés qu'on daigne nous laisser (p. 43). Teboul indique clairement que les positions de Dantin sur les grandes questions sociales et économiques différeront de celles du directeur, Jean-Charles Harvey: elles seront de tendance carrément socialisante, comparées à celles de Harvey qui épouse, lui, de façon quasi inconditionnelle, la cause de la libre entreprise et du laisser-faire (Ibid.). Le premier texte de Dantin à paraître au Jour sera «La complainte d'un chômeur», un poème publié le 16 avril 1938 (p. 4) ${ }^{35}$. Et Dantin se distingue aussi de la plupart des collaborateurs du Jour qui, selon Teboul, s'ils prônent des changements sociaux et culturels, ne préconisent pas néanmoins de grands bouleversements. Réformistes et avant-gardistes, ils ne sont certainement pas révolutionnaires (p. 55). Dantin a en revanche un souci socialiste, une vision collectiviste, et propose un régime populaire révolutionnaire (p. 357). Dantin écrira, en réponse à un rédacteur du Jour qui qualifiait les chômeurs de paresseux:

Ce peuple, [...] vous en avez fait une machine entre vos machines, divisant son travail jusqu'd en faire un réflexe mécanique d'où l'intelligence était presque exclue [...] Vous l'avez rendu dépendant, pour lui et ses enfants, uniquement de vos récompenses. (p. 84)

Le rédacteur en question était un certain Édouard Le Doret, qui affirmait que nul homme n'a le droit divin a l' emploi, pas plus qu'il n'a le droit divin d' habiter Westmount (p. 85). Cette polémique a lieu juste après que Gabrielle Roy fut entrée au Jour comme collaboratrice régulière, et l'on peut croire que ces questions ont pu l'influencer pendant la lente gestation de Bonheur d'occasion.

Le Jour s'attaque aussi au racisme et à l'antisémitisme qui sévissaient au Québec pendant le premier mandat de Maurice Duplessis et se montre ouvert à l'immigration, autre bête noire des nationalistes de droite. Ici encore, il se peut bien que sa collaboration au Jour ait affermi chez Gabrielle Roy sa propre attitude d'ouverture à l'égard des diverses ethnies du pays, issue des expériences de son père comme agent de colonisation dans l'Ouest et fruit de ses premières années à Saint-Boniface-Winnipeg, attitude qui parcourt toute son œuvre ${ }^{36}$. Aussi

35 Selon Victor Teboul, le Jour est le seul journal d'opinion qui, vers la fin des années 30 et au début des années 40, porte un intérêt sérieux et soutenu aux arts et plus particulièrement à la littérature (p. 53). On y remarque de longs articles consacrés à l'art et à ses rapports avec le réel, aux formes esthétiques et aux tendances modernes de l'écriture poétique (p. 55). La littérature y est présentée comme un moyen de persuasion permettant de transformer la société, et un critère - celui de la prise sur le réel - [...] sera fréquemment évoqué dans l'appréciation des auvres (p. 279).

36 Voir notre article «La "généreuse disparité humaine" dans l'cuvre de Gabrielle Roy, de Bonheur d'occasion à la Détresse et l'enchantement», Études canadiennes, XXI, 1986, tome 1, p. 235-244. 
bien en ce qui concerne la littérature que la peinture, les critiques du Jour rejettent la terroiromanie 37 et expriment un désir d'assumer la matiere: l'insistance sur la présence de la ville comme sur celle du corps en témoigne (p. 326) ${ }^{38}$.

Et le domaine de la peinture nous mène directement au second de nos témoins, Henri Girard, qui fut critique d'art au journal libéral le Canada de 1931 à 193939. La première fois que nous avons entendu parler de lui, c'était en lisant un texte intitulé «Les deux sources de l'inspiration: l'imagination et le cœurs $\mathbf{4 0}^{0}$ écrit par l'une des deux sœurs aînées de Gabrielle Roy, Marie-AnnaAdèle Roy, mais apparemment composé par une certaine «Mam'zelle Della» et «trouvé» dans un chalet sur la rivière des Outaouais, du côté du Québec, par une dénommée «Irma Deloy», ces deux prête-nom n'étant évidemment que des anagrammes du nom de l'auteure. Le document est une charge contre l'égoïsme et le total manque de cœur de sa scur Gabrielle. C'est Réginald Hamel, directeur des Archives des lettres canadiennes-françaises de l'Université de Montréal, qui m'a signalé le dépôt dudit document dans son institution et m'en a envoyé une photocopie le 29 octobre 1968. En 1979, paraît un livre de souvenirs sur la famille Roy par la même Marie-Anna A. Roy, le Miroir du passé $^{41}$, qui intègre le gros du tapuscrit original et y ajoute des anecdotes concernant d'autres membres de la famille. L'auteure y a pratiqué de légers changements et coupures par rapport à son texte ronéotypé, nuançant quelque peu - mais pas beaucoup - ses accusations mesquines. Dans l'original, elle avait supprimé les noms des membres de la famille et sa cible principale était désignée par les seuls noms de «Misère» et de «Cad» (sobriquet pour "cadette»), et les dates des événements décrits n'y figuraient pas.

Henri Girard y est désigné comme rédacteur à la Revue moderne de Montréal et commentateur à Radio-Canada, aussi bien que comme journalistepigiste. Adèle Roy lui attribue un rôle primordial dans le développement de la carrière littéraire de Gabrielle Roy. Il est même suggéré dans le tapuscrit que c'est lui qui aurait rédigé le discours de réception de Gabrielle Roy à la Société royale du Canada, bien que dans le livre cette référence ait été enlevée $e^{42}$. Ce qu'a néanmoins gardé Adèle Roy, c'est son insistance à dire que le succès et la réputation de Gabrielle Roy à la suite de la publication de Bonheur d'occasion ont été

37 Ce terme fut employé par Jean-Charles Harvey dans un article du 16 décembre 1939, et est cité par Teboul, p. 319.

38 Berthelot Brunet, cité par Teboul (p. 321-322), y favorise les auteurs iconoclastes, y compris Albert Laberge, qu'il appelle notre Zola, l'as du non-conformisme laurentien.

39 Esther Trépanier, «Crise économique/crise artistique: parallèle ou convergence?», Canadian Issues/Thèmes canadiens, VIII, 1987, p. 181.

40 S.A., ronéotypé, 90 p.

41 Montréal, Québec/Amérique (Littérature d'Amérique).

42 Cad avait commencé à rédiger son discours de réception à la Société royale du Canada [...] - Que je suis fatiguée! Je vais demander à «mon cher ami» de me préparer un discours pour cette réception. («Les deux sources...», p. 62) Voir le Miroir du passé, p. 176: Que je suis fatiguée! Je vais demander à «mon cher ami» de lire et de remanier mon discours de réception [...]. 
le résultat de louches combines: Maintenant célebre grâce au mythe forgé aux trois quarts par la presse à grand tirage, Gabrielle subissait l'assaut des journalistes et des photographes. ${ }^{43}$ Dans «Les deux sources de l'inspiration...», Adèle a intercalé des photocopies de deux lettres qu'elle avait reçues de Henri Girard, lettres qui ne se trouvent pas dans le Miroir du passé. Dans la première, datée du 11 novembre 1945, le critique dit de Gabrielle: Vous savez qu' elle a du génie et qu' elle a écrit un livre immortel. Puis il exprime sa conviction que cette exquise femme de génie ne sera jamais l'auteur d' un seul livre, comme la plupart des écrivains canadiens-français [...]. Ensuite, il donne le détail des arrangements sur lesquels, semble-t-il, lui, Gabrielle et Adèle se sont entendus concemant un projet qu'avaient les deux premiers de trouver auprès [d'Adele] un repaire, le petit coin romantique du bon travail, pour une certaine période de temps ${ }^{44}$.

La seconde lettre est datée du 8 janvier 1948 et fait suite aux noces de Gabrielle et du docteur Marcel Carbotte qui eurent lieu le 26 août 1947 au Manitoba. Adèle avait fait part à Henri Girard de cet événement dans une lettre du 22 décembre 1947, à laquelle il se réfere dans la sienne ${ }^{45}$. Le critique y écrit:

Gabrielle partie, il n'y avait plus de raison de vivre. Songez que pendant sept ans j'ai donné à cette femme qui n'a pas été ma maîtresse le meilleur de mon coeur et de ma pensée. Tout ce qu'il y avait de beau en moi, tout ce que j' avais acquis dans ma jeunesse de sentiments esthétiques, je le lui ai donné sans calcul et sans réserve.

Et Henri Girard d'ajouter.

Remarquez bien, Adèle, que je ne regrette rien. J'ai tout simplement, comme le maître de Mozart, collaboré à l'évolution naturelle d'un génie. Humble collaboration et de peu d'importance en somme, mais qui était nécessaire, alors, pour que l' auvre fût ce qu'elle est.

L'on voit pourquoi Adèle Roy n'a pas cru bon de reproduire cette lettre dans son livre, vu la "cause» qu'elle avait montée de toutes pièces quant au rôle de Henri Girard dans l'œuvre de Gabrielle Roy. Ce rôle, fort apprécié, a été un rôle de mentor, selon Marcel Carbotte ${ }^{46}$, mais il n'a pas été celui que lui attribuait Adèle Roy.

43 Le Miroir du passé, p. 170-171. Voir «Les deux sources...»: Son roman connut dès sa parution un énorme succès de librairie [...]. les journalistes, les critiques, les commentateurs renforcèrent le concert de louanges. (p. 55) Et aussi: Depuis que Cad était devenue une vedette, elle subissait les assauts des journalistes et des photographes. (p. 58)

44 Cette lettre se trouve entre les pages 56 et 57 de «Les deux sources...», et a été écrite sur une feuille portant l'entête de la «Canadian Broadcasting Corporation».

45 Cette lettre suit la page 64 de «Les deux sources...». II semble que les liens étroits entre Gabrielle Roy et Henri Girard se soient établis peu après la rupture entre la romancière et George Wilkinson.

46 Dans une conversation tenue à Québec, le 21 octobre 1988, Marcel Carbotte nous a dit: Henri Girard l'a poussée à écrire. Il l'a adorée et avait pour elle beaucoup d'admiration. Il l'a aidée à maîtriser l'écriture. Le docteur Carbotte nous a aussi signalé que peu après son mariage en 1947, Gabrielle Roy avait brûlé, contre son 
Mais nous nous éloignons quelque peu de notre propos qui est, justement, l'influence qu'a pu avoir Henri Girard sur la vision du monde de Gabrielle Roy, telle qu'exprimée dans son ouvre romanesque. C'est en parcourant les études qu'a consacrées Esther Trépanier à la critique d'art dans le Québec d'entre les deux guerres mondiales ${ }^{47}$ que nous avons retrouvé le nom d'Henri Girard, et que nous avons commencé à réfléchir à une osmose possible. Notons d'abord que Gabrielle Roy fait mention de la Revue moderne aux dernières pages de la Détresse et l'enchantement, où il est question de gagner jusqu'd dix dollars pour une longue nouvelle si je pouvais l'écrire dans le ton qui plaisait a la clientele (p. 504), ce qui contraste avec les trois dollars que lui payait le Jour à peu près au même moment. En fait, ses textes de la Revue moderne furent publiés sur une période bien plus longue que ceux parus dans le Jour entre 1939 et 1940: elle donna régulièrement des textes à ce périodique, où travaillait Henri Girard, entre septembre 1939 et mars 1948, dont bon nombre de nouvelles.

Esther Trépanier considère Girard comme exemplaire des nouvelles attitudes envers l'art èt la culture en général pendant la période qui précède immédiatement la Seconde Guerre mondiale. Dans ses chroniques du Canada, il s'est attaqué à l'académisme autochtone - c'est-à-dire à la représentation du terroir etlou du paysage national comme sujets privilégiés de la «hiérarchie» canadienne des genres ${ }^{48}$. C'était là, selon Trépanier, s'attaquer aussi d une vision du monde bien précise qui était celle d'une grande partie de nos rélites» intellectuelles et politiques (ibid.). En plus de ses critiques d'art, Girard signe des articles sur des sujets variés et ne se prive pas, non plus, pour dénoncer les contradictions du raciste Maurice Duplessis (cité, ibid.). Et Trépanier d'ajouter: Tous les auteurs libéraux ou progressistes de ces années-ld̀ se sont fort heureusement toujours fait un devoir d'attaquer les positions xénophobes souventes fois exprimées par nos chantres de la nation (ibid.). Ainsi, Girard souligne à l'occasion la réception partiale que réservent un Charles Maillard, directeur de l'École des Beaux-Arts, ou un Reynald, de la Presse, à certains peintres du seul fait qu'ils sont étrangers, comme par exemple l'artiste d'origine juive Alexander Bercovitch ou Fritz Brandtner, l'Allemand qui avait fui le nazisme quelques années avant la guerre. Girard est extrêmement reconnaissant (p. 83) à un peintre comme Adrien Hébert, qui privilégie la ville, les installations portuaires et ses travailleurs, et auquel il consacre une dizaine d'articles entre 1931 et 1937, aussi bien qu'à Marc-Aurèle Fortin et ses vues d'Hochelaga, ce quartier ouvrier de l'est montréalais. Trépanier souligne aussi que Girard valorise avec constance [...] des peintres qui

gré à lui, toutes les lettres qu'elle avait reçues de Henri Girard quand elle était à Rawdon dans les Laurentides à rédiger Bonheur d'occasion.

47 Voir la note 44, supra et aussi Esther Trépanier, Peintres juifs et modernité. Jewish Painters and Modernity. Montréal 1930-1945, Montréal, Centre SaidyeBronfman, 1987, p. 73-80; et «L'émergence d'un discours de la modernité dans la critique d'art (Montréal 1918-1938)», dans Yvan Lamonde et Esther Trépanier, l'Avènement de la modernité culturelle au Québec, Québec, Institut québécois de recherche sur la culture, 1986 , p. 82-89.

Esther Trépanier, «L'émergence d'un discours...», p. 82. 
participent d ce mouvement de renouveau du sujet par l'illustration de la vie quotidienne contemporaine [...] (p. 85), et décèle dans ses critiques cette idéologie de «rattrapage» qui deviendra le leitmotiv des générations suivantes (p. 85), jugement que l'on pourrait facilement appliquer aussi à Gabrielle Roy, ainsi que l'on pourrait voir un parallèle entre l'ouverture de Girard envers des artistes de haute qualité de diverses origines ethniques et le portrait des minorités ethniques dans l'œuvre de Gabrielle Roy, auquel nous nous sommes déjà référé.

Néanmoins, écrit Trépanier, Girard ne se laissera pas séduire par ces questionnements qui, da la faveur de la crise économique et de la montée du fascisme, tenteront de redéfinir, dans l'optique d' un engagement politique, la pratique de l'artiste. Et elle ajoute: $A u$ contraire, il n'entend pas que l'art se mêle de la politique, ne serait-ce pour sauver la démocratie (p. 89). Ce rejet de l'engagement artistique est souligné dans le compte rendu que fit Girard d'une exposition tenue à Montréal en mars 1938 au profit de l'CEuvre de secours aux enfants espagnols, c'est-à-dire aux enfants des Républicains victimes de la guerre civile espagnole, exposition à laquelle avaient participé, entre autres, Jean Palardy, Edwin Holgate, John Lyman et Harry Mayerovitch ${ }^{49}$. Dans ce texte, Girard déclare: Je n'entends pas que l'art se mêle des choses de la politique, et précise sa pensée ainsi: $Q u e$ ces artistes-ld soient bolchéviques, fascistes (sic) ou démocrates, le dévouement de leur art à une pensée ou une idée qui n'est pas entièrement de l'ordre de l'esprit désintéressé des choses matérielles reste insupportable. 50

Bien que Gabrielle Roy se soit toujours tenue à l'écart de tout parti ou mouvement politique 51 , nous croyons qu'elle ne partageait pas cette sorte de vision des rapports entre l'art et la réalité, et nous en voulons pour preuve non seulement son œuvre en général, qui est fort éloquente à cet égard, mais en particulier les pages déchirantes qu'elle a écrites dans la Détresse et l'enchantement sur les milliers de réfugiés espagnols qu'elle avait rencontrés en 1939, au village frontalier de Prats-de-Mollo, près de Perpignan. Elle justifie ainsi le titre de l'ouvrage:

Si je m'estime fortunée d'avoir côtoyé assez souvent des gens dont la joie de vivre a rejailli sur moi, il me faut aussi tenir pour un privilège

49 Ce dernier fit des caricatures antifascistes dans le Jour sous la signature de «Henri» à la fin des années trente et pendant les années quarante, selon Esther Trépanier (Peintres juifs..., p. 49).

50 Cité dans Esther Trépanier, «Crise économique...», p. 183.

51 Voir notre Social Realism..., p. 111. Lors d'un entretien que nous avons eu avec lui, Marcel Carbotte a résumé ainsi le credo de Gabrielle Roy: L'auteur n'a d'engagement qu'à la liberté et à la vérité. Marie-Anna A. Roy prétend, dans le Miroir du passé, que sa scur Gabrielle lui parla avec enthousiasme du «Red Dean» [Hewlett Johnson], ce doyen rouge d'Angleterre qui prêchait le marxisme (p. 142). Mais il n'y a pas de preuves qu'elle ait pris une telle position politique. Gabrielle Roy nous dit lors d'une entrevue en 1966 que, tout au plus, elle fréquenta un groupe d'étude sous l'égide du Syndicat uni des travailleurs du textile pendant les années quarante (le docteur Carbotte me dit que Gabrielle Roy admirait le courage de Madeleine Parent, leader de ce même syndicat). A l'époque de Bonheur 
— très haut et très douloureux - d' avoir approché quelquefois le plus grand malheur du monde. [...] Ah Dieu! Le spectacle que j'eus sous les yeux, dont le souvenir hante encore mes nuits avec des fragments d' horreur comme dans Guernica! [...] Dans ma chambre glaciale [...] je me lançai à écrire mes premières pages dictées par l'indignation, la pitié, la grande souffrance d'appartenir à l'espèce humaine. [...] Je finis [...] par l'envoyer avec quelques photos à la Presse de Montréal. Ces quelques pages, sous une signature inconnue, sympathiques à l'Espagne rouge à l'heure où a Montréal même Malraux $n$ 'avait pu obtenir l'autorisation de se porter en public à sa défense $e^{52}$,

d'occasion j'étais peut-être à demi révolutionnaire, ajouta-t-elle. Antoine Sirois nous a signalé que, lors de la rédaction de son article sur Bonheur d'occasion pour le tome III du Dictionnaire des æuvres littéraires du Québec, il avait rencontré Gabrielle Roy qui lui fit part d'une rencontre qu'elle avait sollicitée avec Madeleine Parent dans les années 1940 (les archives Gabrielle Roy de la Bibliothèque nationale du Canada contiennent aussi une lettre à la romancière d'un autre leader du Syndicat des ouvriers du textile, Charles Lipton, auteur d'une histoire du syndicalisme canadien, traduite en français et publiée aux éditions Parti pris. Malheureusement, au moment de la rédaction de cette note, Ima Larouche, responsable de la collection Gabrielle Roy à la B.N.C., ne pouvait retrouver ladite lettre. Le docteur Carbotte nous a aussi parlé de l'amitié entre sa femme et un syndicaliste anglophone du textile qui était sans doute M. Lipton). Dans son texte du DOLQ, Antoine Sirois écrit que Gabrielle Roy, bien que sensible à la pensée socialiste de Jaurès ou du syndicaliste [Léon] Jouhaux [chêf du Congrès général du travail en France de 1909 à 1947, alors qu'il rompit avec les communistes pour créer, un peu plus tard, la centrale Force ouvrière], rejette la violence et refuse comme écrivain de s'embrigader dans un mouvement (p. 132). Réjean Robidoux nous a envoyé un article intitulé "Gabrielle Roy ou la condition humaine», paru dans le Droit d'Ottawa du 23 juillet 1983 (p. 6) et signé Fulgence Charpentier, où il est question de l'amitie entre la romancière et Grace Woodsworth MacInnis, fille du fondateur du C.C.F., J.S. Woodsworth, et femme du député fédéral Angus McInnis du C.C.F. et du Nouveau Parti démocratique, et elle-même députée du N.P.D. à la Chambre des communes de 1965 à 1974. Les deux jeunes femmes se voyaient souvent, soit à Ottawa, soit à Winnipeg, deux âmes faites pour se comprendre, écrit Charpentier. Sous ses apparences timides et réservées, la future romancière se passionna pour les idées nouvelles et les combats qui se livraient sur la place publique contre les injustices et les abus de pouvoir. Lors d'une conversation téléphonique, le 5 janvier 1989 , M. Charpentier nous raconta qu'il avait fait la connaissance de Gabrielle Roy à Ottawa lors de la participation du Cercle Molière aux finales du concours Bessborough (Festival d'art dramatique canadien) pendant les années 1930; il la revit, ainsi que son mari, à Paris à la fin des années 1940. M. Charpentier était, à cette époque, secrétaire à l'ambassade du Canada, chargé des affaires culturelles et des relations avec la presse. Il faudrait toutefois nuancer certains propos de M. Charpentier, selon qui Gabrielle Roy a été influencée par la grève générale de Winnipeg en 1919 (elle n'avait que dix ans), dont un des leaders emprisonnés fut justement $\mathbf{M}$. Woodsworth. M. Charpentier écrit là-dessus: Comment rester indifférente dans une lutte qui transformait sa ville en champ de bataille pour une cause qui lui paraissait juste puisqu'il s'agissait du sort malheureux du faible contre la force?

52 Voir à ce sujet le discours tenu par le cardinal Rodrigue Villeneuve à l'occasion de la visite d'André Malraux au Québec en 1937, où le prélat soutint les étudiants qui avaient empêché l'écrivain français de prononcer un discours à Montréal, alléguant 
j'imagine avec quelle alacrité celui qui les a lues a dû les envoyer rouler dans le panier. (p. 487-490)

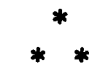

En guise de conclusion, rappelons-nous donc ce tout ce que j'aurais acquis de ressources, de connaissances de l' humain dont parle Gabrielle Roy vers la fin de la Détresse et l'enchantement, au moment où elle évoque la gestation de son premier roman. N'est-ce pas que ces ressources et connaissances venaient, certes, de sa propre formation d'être humain combien sensible et indépendant, mais, sans doute, aussi de ces moments de sa vie qu'elle a partagés avec George Wilkinson et Henri Girard?

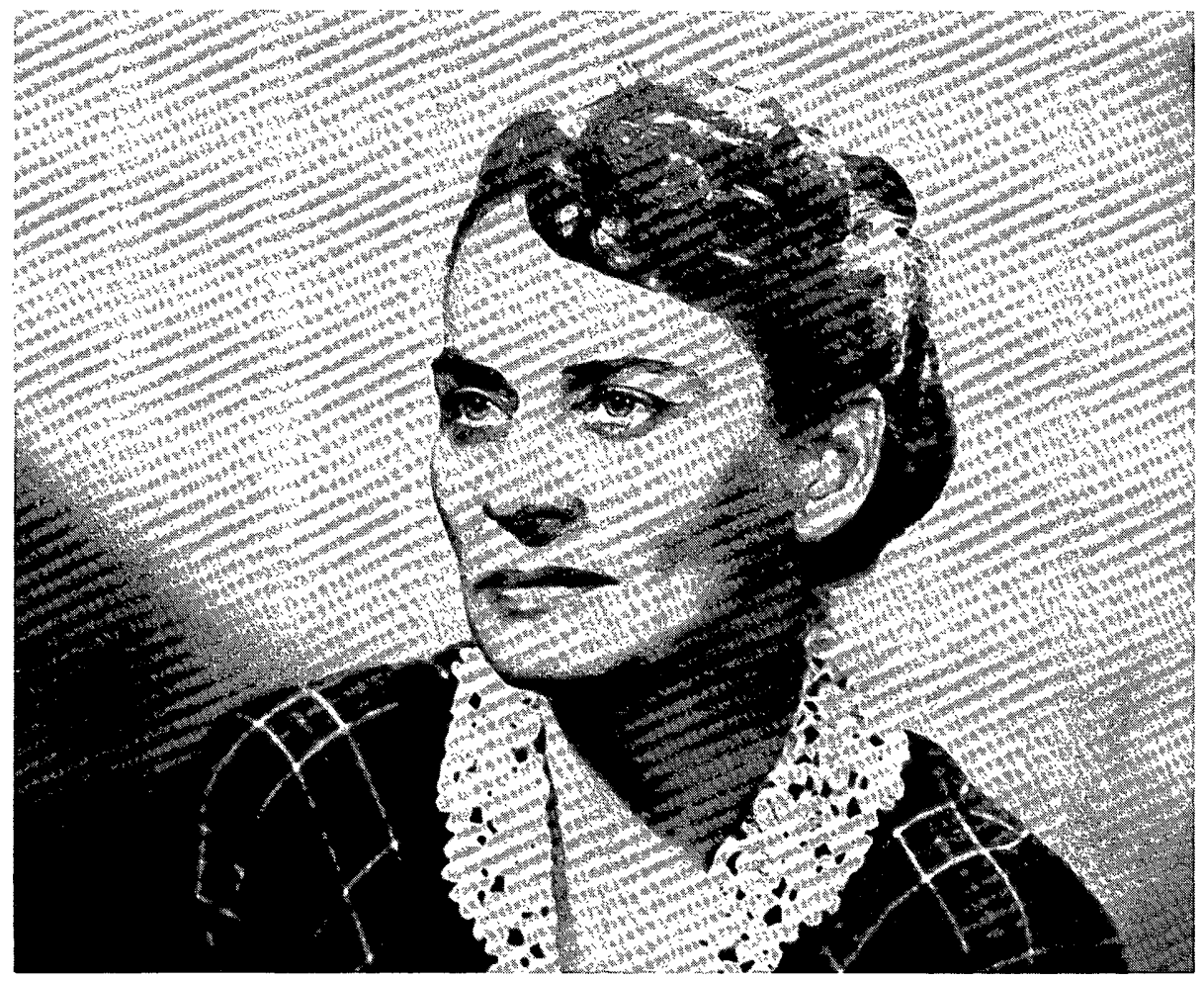

que avant la loi, il y a le droit de la nature [...] Sous le prétexte de respecter une démocratie moribonde, on agite le fantôme d'un fascisme illusoire [...] (l'Action catholique, 2 novembre 1937, p. 4). 